\title{
APPLICATIONS OF A DIFFERENTIAL SUBORDINATION INVOLVING THE CONVOLUTION OF ANALYTIC FUNCTIONS
}

\author{
R. Brar AND S. S. Billing
}

AbSTRACT. In the present paper, we derive a subordination theorem involving the convolution of analytic functions. As special cases of our main results, we obtain the sufficient conditions for analytic functions to be parabolic $\phi$-like, parabolic starlike, $\phi$-like and starlike.

2010 Mathematics Subject Classification: 30C45, 30C80.

Keywords: analytic function, univalent function, differential subordination, parabolic $\phi-$ like function, parabolic starlike function, $\phi-$ like function, starlike function, convolution.

\section{INTRODUCTION}

Let $\mathcal{A}$ denote the class of all functions $f$ analytic in $\mathbb{E}=\{z:|z|<1\}$, normalized by the conditions $f(0)=f^{\prime}(0)-1=0$. Therefore, Taylor's series expansion of $f \in \mathcal{A}$, is given by

$$
f(z)=z+\sum_{k=2}^{\infty} a_{k} z^{k} .
$$

Let the functions $f$ and $g$ be analytic in $\mathbb{E}$. We say that $f$ is subordinate to $g$ written as $f \prec g$ in $\mathbb{E}$, if there exists a Schwarz function $\phi$ in $\mathbb{E}$ (i.e. $\phi$ is regular in $|z|<1, \phi(0)=0$ and $|\phi(z)| \leq|z|<1)$ such that

$$
f(z)=g(\phi(z)), \quad|z|<1 .
$$

Let $\Phi: \mathbb{C}^{2} \times \mathbb{E} \rightarrow \mathbb{C}$ be an analytic function, $p$ an analytic function in $\mathbb{E}$, with $\left(p(z), z p^{\prime}(z) ; z\right) \in \mathbb{C}^{2} \times \mathbb{E}$ for all $z \in \mathbb{E}$ and $h$ be univalent in $\mathbb{E}$. Then the function $p$ is said to satisfy first order differential subordination if

$$
\Phi\left(p(z), z p^{\prime}(z) ; z\right) \prec h(z), \quad \Phi(p(0), 0 ; 0)=h(0) .
$$


R. Brar and S. S. Billing - Applications of a Differential Subordination ...

A univalent function $q$ is called a dominant of the differential subordination (1) if $p(0)=q(0)$ and $p \prec q$ for all $p$ satisfying (1). A dominant $\tilde{q}$ that satisfies $\tilde{q} \prec q$ for all dominants $q$ of (1), is said to be the best dominant of (1). The best dominant is unique up to a rotation of $\mathbb{E}$.

Let $f(z)=\sum_{k=0}^{\infty} a_{k} z^{k}$ and $g(z)=\sum_{k=0}^{\infty} b_{k} z^{k}$ be two analytic functions, then convolution of $f(z)$ and $g(z)$, written as $(f * g)(z)$ is defined by

$$
(f * g)(z)=\sum_{k=0}^{\infty} a_{k} b_{k} z^{k} .
$$

A function $f \in \mathcal{A}$ is said to be starlike of order $\alpha(0 \leq \alpha<1)$ in $\mathbb{E}$ if

$$
\Re\left(\frac{z f^{\prime}(z)}{f(z)}\right)>\alpha, z \in \mathbb{E} .
$$

Let $\mathcal{S}^{*}(\alpha)$ denote the class of starlike functions of order $\alpha$. Write $\mathcal{S}^{*}(0)=\mathcal{S}^{*}$, the class of starlike functions.

A function $f \in \mathcal{A}$ is said to be parabolic starlike in $\mathbb{E}$, if

$$
\Re\left(\frac{z f^{\prime}(z)}{f(z)}\right)>\left|\frac{z f^{\prime}(z)}{f(z)}-1\right|, z \in \mathbb{E} .
$$

The class of parabolic starlike functions is denoted by $\mathcal{S}_{\mathcal{P}}$.

A function $f \in \mathcal{A}$ is said to be strongly starlike of order $\alpha, 0<\alpha \leq 1$, if

$$
\left|\arg \frac{z f^{\prime}(z)}{f(z)}\right|<\frac{\alpha \pi}{2}, z \in \mathbb{E} .
$$

or, equivalently

$$
\frac{z f^{\prime}(z)}{f(z)} \prec\left(\frac{1+z}{1-z}\right)^{\alpha}, z \in \mathbb{E} .
$$

Let $\tilde{\mathcal{S}}(\alpha)$ denote the class of strongly starlike functions of order $\alpha$.

Let $\phi$ be analytic in a domain containing $f(\mathbb{E}), \phi(0)=0$ and $\Re\left(\phi^{\prime}(0)\right)>0$. Then, the function $f \in \mathcal{A}$ is said to be $\phi$-like in $\mathbb{E}$ if

$$
\Re\left(\frac{z f^{\prime}(z)}{\phi(f(z))}\right)>0, \quad z \in \mathbb{E} .
$$

This concept was introduced by L. Brickman [1]. He proved that an analytic function $f \in \mathcal{A}$ is univalent if and only if $f$ is $\phi$-like for some $\phi$. Later, Ruscheweyh [6] investigated the following general class of $\phi$-like functions: 
Let $\phi$ be analytic in a domain containing $f(\mathbb{E})$, where $\phi(0)=0, \phi^{\prime}(0)=1$ and $\phi(w) \neq 0$ for some $w \in f(\mathbb{E}) \backslash\{0\}$. Let $q(z)$ be a fixed analytic function in $\mathbb{E}, q(0)=1$. Then the function $f \in \mathcal{A}$ is called $\phi$-like with respect to $q$, if

$$
\frac{z f^{\prime}(z)}{\phi(f(z))} \prec q(z), \quad z \in \mathbb{E} .
$$

A function $f \in \mathcal{A}$ is said to be parabolic $\phi$-like in $\mathbb{E}$ if

$$
\Re\left(\frac{z f^{\prime}(z)}{\phi(f(z))}\right)>\left|\frac{z f^{\prime}(z)}{\phi(f(z))}-1\right|, \quad z \in \mathbb{E} .
$$

Define the parabolic domain $\Omega$ as under:

$$
\Omega=\left\{u+i v: u>\sqrt{(u-1)^{2}+v^{2}}\right\} .
$$

Note that the conditions (2) and (4) are equivalent to the condition that $\frac{z f^{\prime}(z)}{f(z)}$ and $\frac{z f^{\prime}(z)}{\phi(f(z))}$ take values in the parabolic domain $\Omega$ respectively.

Ronning [5] and Ma and Minda [2] showed that the function defined by

$$
q(z)=1+\frac{2}{\pi^{2}}\left(\log \left(\frac{1+\sqrt{z}}{1-\sqrt{z}}\right)\right)^{2}
$$

maps the unit disk $\mathbb{E}$ onto the parabolic domain $\Omega$. Therefore, the condition (2) and (4) are equivalent to following conditions respectively:

$$
\frac{z f^{\prime}(z)}{f(z)} \prec q(z), \quad z \in \mathbb{E}
$$

and

$$
\frac{z f^{\prime}(z)}{\phi(f(z))} \prec q(z), \quad z \in \mathbb{E}
$$

where $\mathrm{q}(\mathrm{z})$ is given by $(5)$.

In 2005, Ravichandran et al. [4] proved the following result for $\phi-$ like functions.

Theorem 1. Let $\alpha \neq 0$ be a complex number and $q(z)$ be convex univalent in $\mathbb{E}$. Define $h(z)=\alpha q^{2}(z)+(1-\alpha) q(z)+\alpha z q^{\prime}(z)$. Further assume that $\Re\left\{\frac{1-\alpha}{\alpha}+2 q(z)+\right.$ $\left.\left(1+\frac{z q^{\prime \prime}(z)}{q^{\prime}(z)}\right)\right\}>0, \quad z \in \mathbb{E}$. If $f \in \mathbb{A}$ satisfies

$$
\frac{z f^{\prime}(z)}{\phi(f(z))}\left[1+\frac{\alpha z f^{\prime \prime}(z)}{f^{\prime}(z)}+\frac{\alpha z\left[f^{\prime}(z)-\{\phi(f(z))\}^{\prime}\right]}{\phi(f(z))}\right] \prec h(z)
$$


R. Brar and S. S. Billing - Applications of a Differential Subordination ...

then

$$
\frac{z f^{\prime}(z)}{\phi(f(z))} \prec q(z)
$$

and $q(z)$ is the best dominant.

Later, Shanmugham et al. [7] obtained the following result.

Theorem 2. Let $q(z) \neq 0$ be analytic and univalent in $\mathbb{E}$ with $q(0)=1$ such that $\frac{z q^{\prime}(z)}{q(z)}$ is starlike univalent in $\mathbb{E}$. Let $q(z)$ satisfy

$$
\Re\left[1+\frac{\alpha q(z)}{\gamma}-\frac{z q^{\prime}(z)}{q(z)}+\frac{z q^{\prime \prime}(z)}{q^{\prime}(z)}\right] \geq 0 .
$$

Let for $f, g \in \mathcal{A}$

$$
\Psi(\alpha, \gamma, g ; z):=\alpha\left\{\frac{z(f * g)^{\prime}(z)}{\Phi(f * g)(z)}\right\}+\gamma\left\{1+\frac{z(f * g)^{\prime \prime}(z)}{(f * g)^{\prime}(z)}-\frac{z(\Phi(f * g)(z))^{\prime}}{\Phi(f * g)(z)}\right\} .
$$

If $q$ satisfies

$$
\Psi(\alpha, \gamma, g ; z) \prec \alpha q(z)+\frac{\gamma z q^{\prime}(z)}{q(z)}
$$

then

$$
\frac{z(f * g)^{\prime}(z)}{\Phi(f * g)(z)} \prec q(z)
$$

and $q(z)$ is the best dominant.

In the present paper, we derive a subordination theorem involving the convolution of analytic functions. As special cases of our main results, we obtain the sufficient conditions for analytic functions to be parabolic $\phi$-like, parabolic starlike, $\phi$-like and starlike.

\section{PRELIMINARIES}

To prove our main results, we shall use the following lemma of Miller and Mocanu ([3], Theorem 3.4h, p.132).

Lemma 3. Let $q$ be a univalent in $\mathbb{E}$ and let $\Theta$ and $\Phi$ be analytic in a domain $\mathbb{D}$ containing $q(\mathbb{E})$, with $\Phi(w) \neq 0$, when $w \in q(\mathbb{E})$. Set $Q(z)=z q^{\prime}(z) \Phi[q(z)], \quad h(z)=$ $\Theta[q(z)]+Q(z)$ and suppose that either

(i) $h$ is convex, or

(ii) $Q$ is starlike. 
In addition, assume that

(iii) $\Re \frac{z h^{\prime}(z)}{Q(z)}=\Re\left[\frac{\Theta^{\prime}[q(z)]}{\Phi[q(z)]}+\frac{z Q^{\prime}(z)}{Q(z)}\right]>0$.

If $p$ is analytic in $\mathbb{E}$, with $p(0)=q(0), p(\mathbb{E}) \subset \mathbb{D}$ and

$$
\Theta[p(z)]+z p^{\prime}(z) \Phi[p(z)] \prec \Theta[q(z)]+z q^{\prime}(z) \Phi[q(z)]=h(z),
$$

then $p \prec q$, and $q$ is the best dominant.

\section{Main Results}

In what follows, all the powers taken are principal ones.

Theorem 4. Let $q(z) \neq 0$, be a univalent function in $\mathbb{E}$ such that (i) $\Re\left[1+\frac{z q^{\prime \prime}(z)}{q^{\prime}(z)}+(\gamma-1) \frac{z q^{\prime}(z)}{q(z)}\right]>0$ and

(ii) $\Re\left[1+\frac{z q^{\prime \prime}(z)}{q^{\prime}(z)}+(\gamma-1) \frac{z q^{\prime}(z)}{q(z)}+\frac{\beta(1-\alpha)}{\alpha}(q(z))^{\beta-\gamma}+\gamma\right]>0$.

If $f$ and $g \in \mathcal{A}$ satisfy

$$
\begin{gathered}
(1-\alpha)\left[\frac{z(f * g)^{\prime}(z)}{\phi(f * g)(z)}\right]^{\beta}+\alpha\left[\frac{z(f * g)^{\prime}(z)}{\phi(f * g)(z)}\right]^{\gamma}\left[2+\frac{z(f * g)^{\prime \prime}(z)}{(f * g)^{\prime}(z)}-\frac{z(\phi((f * g)(z)))^{\prime}}{\phi((f * g)(z))}\right] \\
\prec(1-\alpha)(q(z))^{\beta}+\alpha(q(z))^{\gamma}\left(1+\frac{z q^{\prime}(z)}{q(z)}\right)
\end{gathered}
$$

then

$$
\frac{z(f * g)^{\prime}(z)}{\phi((f * g)(z))} \prec q(z), \quad z \in \mathbb{E},
$$

where $\alpha, \beta, \gamma$ are complex numbers such that $\alpha \neq 0$, and $q(z)$ is the best dominant.

Proof. Define the function $p(z)$ by

$$
p(z)=\frac{z(f * g)^{\prime}(z)}{\phi(f * g)(z)}, \quad z \in \mathbb{E} .
$$

Then the function $p(z)$ is analytic in $\mathbb{E}$ and $p(0)=1$. Therefore, from equation (6) we obtain:

$$
(1-\alpha)(p(z))^{\beta}+\alpha(p(z))^{\gamma}\left(1+\frac{z p^{\prime}(z)}{p(z)}\right) \prec(1-\alpha)(q(z))^{\beta}+\alpha(q(z))^{\gamma}\left(1+\frac{z q^{\prime}(z)}{q(z)}\right),
$$

Let us define the functions $\Theta$ and $\Phi$ as follows:

$$
\Theta(w)=(1-\alpha) w^{\beta}+\alpha w^{\gamma}
$$


R. Brar and S. S. Billing - Applications of a Differential Subordination ...

and

$$
\Phi(w)=\alpha w^{\gamma-1}
$$

Obviously, both the functions $\Theta$ and $\Phi$ are analytic in $\mathbb{D}=\mathbb{C} \backslash\{0\}$ and $\Phi(w) \neq 0$ in $\mathbb{D}$. Therefore,

$$
Q(z)=\Phi(q(z)) z q^{\prime}(z)=\alpha z q^{\prime}(z)(q(z))^{\gamma-1}
$$

and

$$
h(z)=\Theta(q(z))+Q(z)=(1-\alpha)(q(z))^{\beta}+\alpha(q(z))^{\gamma}\left(1+\frac{z q^{\prime}(z)}{q(z)}\right) .
$$

On differentiating, we obtain $\frac{z Q^{\prime}(z)}{Q(z)}=1+\frac{z q^{\prime \prime}(z)}{q^{\prime}(z)}+(\gamma-1) \frac{z q^{\prime}(z)}{q(z)}$ and

$$
\frac{z h^{\prime}(z)}{Q(z)}=1+\frac{z q^{\prime \prime}(z)}{q^{\prime}(z)}+(\gamma-1) \frac{z q^{\prime}(z)}{q(z)}+\frac{\beta(1-\alpha)}{\alpha}(q(z))^{\beta-\gamma}+\gamma
$$

In view of the given conditions, we see that $\mathrm{Q}$ is starlike and $\Re\left(\frac{z h^{\prime}(z)}{Q(z)}\right)>0$.

Therefore, the proof, now follows from Lemma 3.

Selecting $g(z)=\frac{z}{1-z}$ in Theorem 4, we get the following result:

Theorem 5. Let $q(z) \neq 0$, be a univalent function in $\mathbb{E}$ such that (i) $\Re\left[1+\frac{z q^{\prime \prime}(z)}{q^{\prime}(z)}+(\gamma-1) \frac{z q^{\prime}(z)}{q(z)}\right]>0$ and

(ii) $\Re\left[1+\frac{z q^{\prime \prime}(z)}{q^{\prime}(z)}+(\gamma-1) \frac{z q^{\prime}(z)}{q(z)}+\frac{\beta(1-\alpha)}{\alpha}(q(z))^{\beta-\gamma}+\gamma\right]>0$.

If $f \in \mathcal{A}$ satisfy

$$
\begin{aligned}
(1-\alpha)\left[\frac{z f^{\prime}(z)}{\phi(f(z))}\right]^{\beta}+\alpha & {\left[\frac{z f^{\prime}(z)}{\phi(f(z))}\right]^{\gamma}\left[2+\frac{z f^{\prime \prime}(z)}{f^{\prime}(z)}-\frac{z\left(\phi(f(z))^{\prime}\right.}{\phi(f(z))}\right] } \\
& \prec(1-\alpha)(q(z))^{\beta}+\alpha(q(z))^{\gamma}\left(1+\frac{z q^{\prime}(z)}{q(z)}\right),
\end{aligned}
$$

then

$$
\frac{z f^{\prime}(z)}{\phi(f(z))} \prec q(z), \quad z \in \mathbb{E},
$$

where $\alpha, \beta, \gamma$ are complex numbers such that $\alpha \neq 0$, and $q(z)$ is the best dominant. Selecting $\phi(w)=w$ in Theorem 5, we get: 
R. Brar and S. S. Billing - Applications of a Differential Subordination ...

Theorem 6. Let $q(z) \neq 0$, be a univalent function in $\mathbb{E}$ such that (i) $\Re\left[1+\frac{z q^{\prime \prime}(z)}{q^{\prime}(z)}+(\gamma-1) \frac{z q^{\prime}(z)}{q(z)}\right]>0$ and

(ii) $\Re\left[1+\frac{z q^{\prime \prime}(z)}{q^{\prime}(z)}+(\gamma-1) \frac{z q^{\prime}(z)}{q(z)}+\frac{\beta(1-\alpha)}{\alpha} q(z)^{\beta-\gamma}+\gamma\right]>0$.

If $f \in \mathcal{A}$ satisfy

$$
\begin{aligned}
(1-\alpha)\left[\frac{z f^{\prime}(z)}{f(z)}\right]^{\beta}+ & \alpha\left[\frac{z f^{\prime}(z)}{f(z)}\right]^{\gamma}\left[2+\frac{z f^{\prime \prime}(z)}{f^{\prime}(z)}-\frac{z f^{\prime}(z)}{f(z)}\right] \\
& \prec(1-\alpha)(q(z))^{\beta}+\alpha(q(z))^{\gamma}\left(1+\frac{z q^{\prime}(z)}{q(z)}\right),
\end{aligned}
$$

then

$$
\frac{z f^{\prime}(z)}{f(z)} \prec q(z), \quad z \in \mathbb{E},
$$

where $\alpha, \beta, \gamma$ are complex numbers such that $\alpha \neq 0$, and $q(z)$ is the best dominant.

\section{Applications}

Remark 1. When we select $\beta=1, \gamma=0$ and $q(z)=1+\frac{2}{\pi^{2}}\left(\log \left(\frac{1+\sqrt{z}}{1-\sqrt{z}}\right)\right)^{2}$ in Theorem 5 and Theorem 6, a little calculation yields that

$$
1+\frac{z q^{\prime \prime}(z)}{q^{\prime}(z)}-\frac{z q^{\prime}(z)}{q(z)}=\frac{1+z}{2(1-z)}+\frac{\sqrt{z}}{(1-z) \log \left(\frac{1+\sqrt{z}}{1-\sqrt{z}}\right)}-\frac{\frac{4 \sqrt{z}}{\pi^{2}(1-z)} \log \left(\frac{1+\sqrt{z}}{1-\sqrt{z}}\right)}{1+\frac{2}{\pi^{2}}\left(\log \left(\frac{1+\sqrt{z}}{1-\sqrt{z}}\right)\right)^{2}}
$$

and

$$
\begin{gathered}
1+\frac{z q^{\prime \prime}(z)}{q^{\prime}(z)}-\frac{z q^{\prime}(z)}{q(z)}+\left(\frac{1-\alpha}{\alpha}\right) q(z)=\frac{1+z}{2(1-z)}+\frac{\sqrt{z}}{(1-z) \log \left(\frac{1+\sqrt{z}}{1-\sqrt{z}}\right)}-\frac{\frac{4 \sqrt{z}}{\pi^{2}(1-z)} \log \left(\frac{1+\sqrt{z}}{1-\sqrt{z}}\right)}{1+\frac{2}{\pi^{2}}\left(\log \left(\frac{1+\sqrt{z}}{1-\sqrt{z}}\right)\right)^{2}} \\
+\frac{(1-\alpha)}{\alpha}\left(1+\frac{2}{\pi^{2}}\left(\log \left(\frac{1+\sqrt{z}}{1-\sqrt{z}}\right)\right)^{2}\right) .
\end{gathered}
$$

Thus for real number $\alpha$ such that $0<\alpha \leq 1$, we notice that $q(z)$ satisfies the condition (i) and (ii) in Theorem 5 and Theorem 6. Therefore, we derive next two results from Theorem 5 and Theorem 6 respectively. 
R. Brar and S. S. Billing - Applications of a Differential Subordination ...

Theorem 7. Let $\alpha$ be a real number such that $0<\alpha \leq 1$. If $f \in \mathcal{A}$ satisfies

$$
\begin{gathered}
(1-\alpha) \frac{z f^{\prime}(z)}{\phi(f(z))}+\alpha \\
\left(1+\frac{z f^{\prime \prime}(z)}{f^{\prime}(z)}-\frac{z\left(\phi(f(z))^{\prime}\right.}{\phi(f(z))}\right) \\
\prec(1-\alpha)\left[1+\frac{2}{\pi^{2}}\left(\log \left(\frac{1+\sqrt{z}}{1-\sqrt{z}}\right)\right)^{2}\right] \\
+\frac{\frac{4 \alpha \sqrt{z}}{\pi^{2}(1-z)} \log \left(\frac{1+\sqrt{z}}{1-\sqrt{z}}\right)}{1+\frac{2}{\pi^{2}}\left(\log \left(\frac{1+\sqrt{z}}{1-\sqrt{z}}\right)\right)^{2}},
\end{gathered}
$$

then

$$
\frac{z f^{\prime}(z)}{\phi(f(z))} \prec 1+\frac{2}{\pi^{2}}\left(\log \left(\frac{1+\sqrt{z}}{1-\sqrt{z}}\right)\right)^{2}, \quad z \in \mathbb{E} .
$$

Theorem 8. Let $\alpha$ be a real number such that $0<\alpha \leq 1$. If $f \in \mathcal{A}$ satisfies

$$
\begin{gathered}
(1-\alpha) \frac{z f^{\prime}(z)}{f(z)}+\alpha\left(1+\frac{z f^{\prime \prime}(z)}{f^{\prime}(z)}-\frac{z f^{\prime}(z)}{f(z)}\right) \\
\prec(1-\alpha)\left[1+\frac{2}{\pi^{2}}\left(\log \left(\frac{1+\sqrt{z}}{1-\sqrt{z}}\right)\right)^{2}\right] \\
+\frac{\frac{4 \alpha \sqrt{z}}{\pi^{2}(1-z)} \log \left(\frac{1+\sqrt{z}}{1-\sqrt{z}}\right)}{1+\frac{2}{\pi^{2}}\left(\log \left(\frac{1+\sqrt{z}}{1-\sqrt{z}}\right)\right)^{2}}
\end{gathered}
$$

then

$$
\frac{z f^{\prime}(z)}{f(z)} \prec 1+\frac{2}{\pi^{2}}\left(\log \left(\frac{1+\sqrt{z}}{1-\sqrt{z}}\right)\right)^{2}, \quad z \in \mathbb{E} .
$$

Remark 2. When we select $\beta=1, \gamma=0$ and $q(z)=e^{z}$ in Theorem 5 and Theorem 6 , a little calculation yields that

$$
1+\frac{z q^{\prime \prime}(z)}{q^{\prime}(z)}-\frac{z q^{\prime}(z)}{q(z)}=1
$$

and

$$
1+\frac{z q^{\prime \prime}(z)}{q^{\prime}(z)}-\frac{z q^{\prime}(z)}{q(z)}+\left(\frac{1-\alpha}{\alpha}\right) q(z)=1+\left(\frac{1-\alpha}{\alpha}\right) e^{z} .
$$

Thus for positive real number $\alpha$ such that $0<\alpha \leq 1$, we notice that $q(z)$ satisfies the condition (i) and (ii) in Theorem 5 and Theorem 6. Therefore, we get the following results from Theorem 5 and Theorem 6 respectively. 
R. Brar and S. S. Billing - Applications of a Differential Subordination ...

Theorem 9. Let $\alpha$ be a real number such that $0<\alpha \leq 1$. If $f \in \mathcal{A}$ satisfies

$$
\begin{array}{r}
(1-\alpha) \frac{z f^{\prime}(z)}{\phi(f(z))}+\alpha\left(1+\frac{z f^{\prime \prime}(z)}{f^{\prime}(z)}-\frac{z\left(\phi(f(z))^{\prime}\right.}{\phi(f(z))}\right) \\
\prec(1-\alpha) e^{z}+\alpha z,
\end{array}
$$

then

$$
\frac{z f^{\prime}(z)}{\phi(f(z))} \prec e^{z}, \quad z \in \mathbb{E} .
$$

Theorem 10. Let $\alpha$ be a real number such that $0<\alpha \leq 1$. If $f \in \mathcal{A}$ satisfies

$$
\begin{array}{r}
(1-\alpha) \frac{z f^{\prime}(z)}{f(z)}+\alpha\left(1+\frac{z f^{\prime \prime}(z)}{f^{\prime}(z)}-\frac{z f^{\prime}(z)}{f(z)}\right) \\
\prec(1-\alpha) e^{z}+\alpha z,
\end{array}
$$

then

$$
\frac{z f^{\prime}(z)}{f(z)} \prec e^{z}, \quad z \in \mathbb{E}
$$

Remark 3. When we select $\beta=1, \gamma=0$ and $q(z)=\frac{1+(1-2 \delta) z}{1-z} ; 0 \leq \delta<1$ in Theorem 5 and Theorem 6, a little calculation yields that

$$
1+\frac{z q^{\prime \prime}(z)}{q^{\prime}(z)}-\frac{z q^{\prime}(z)}{q(z)}=\frac{1+(1-2 \delta) z^{2}}{(1-z)(1+(1-2 \delta) z)}
$$

and

$1+\frac{z q^{\prime \prime}(z)}{q^{\prime}(z)}-\frac{z q^{\prime}(z)}{q(z)}+\left(\frac{1-\alpha}{\alpha}\right) q(z)=\frac{1+(1-2 \delta) z^{2}}{(1-z)(1+(1-2 \delta) z)}+\left(\frac{1-\alpha}{\alpha}\right)\left[\frac{1+(1-2 \delta) z}{1-z}\right]$.

Thus for real number $\alpha$ such that $0<\alpha \leq 1$, we notice that $q(z)$ satisfies the condition (i) and (ii) in Theorem 5 and Theorem 6. Therefore, we arrive at the following results from Theorem 5 and Theorem 6 respectively.

Theorem 11. Let $\alpha$ be a real number such that $0<\alpha \leq 1$. If $f \in \mathcal{A}$ satisfies

$$
\begin{aligned}
& (1-\alpha) \frac{z f^{\prime}(z)}{\phi(f(z))}+\alpha\left(1+\frac{z f^{\prime \prime}(z)}{f^{\prime}(z)}-\frac{z\left(\phi(f(z))^{\prime}\right.}{\phi(f(z))}\right) \\
& \prec(1-\alpha)\left[\frac{1+(1-2 \delta) z}{1-z}\right]+\frac{2 \alpha z(1-\delta)}{(1-z)[1+(1-2 \delta) z]}, z \in \mathbb{E},
\end{aligned}
$$

then

$$
\frac{z f^{\prime}(z)}{\phi(f(z))} \prec \frac{1+(1-2 \delta) z}{1-z} \text {, where } 0 \leq \delta<1 \text {. }
$$


R. Brar and S. S. Billing - Applications of a Differential Subordination ...

Theorem 12. Let $\alpha$ be a real number such that $0<\alpha \leq 1$. If $f \in \mathcal{A}$ satisfies

$$
\begin{aligned}
& (1-\alpha) \frac{z f^{\prime}(z)}{f(z)}+\alpha\left(1+\frac{z f^{\prime \prime}(z)}{f^{\prime}(z)}-\frac{z f^{\prime}(z)}{f(z)}\right) \\
& \prec(1-\alpha)\left[\frac{1+(1-2 \delta) z}{1-z}\right]+\frac{2 \alpha z(1-\delta)}{(1-z)[1+(1-2 \delta) z]}, z \in \mathbb{E},
\end{aligned}
$$

then

$$
\frac{z f^{\prime}(z)}{f(z)} \prec \frac{1+(1-2 \delta) z}{1-z} \text {, where } 0 \leq \delta<1 \text {. }
$$

Remark 4. When we select $\beta=1, \gamma=0$ and $q(z)=1+a z ; 0 \leq a<1$ in Theorem 5 and Theorem 6, a little calculation yields that

$$
1+\frac{z q^{\prime \prime}(z)}{q^{\prime}(z)}-\frac{z q^{\prime}(z)}{q(z)}=\frac{1}{1+a z}
$$

and

$$
1+\frac{z q^{\prime \prime}(z)}{q^{\prime}(z)}-\frac{z q^{\prime}(z)}{q(z)}+\left(\frac{1-\alpha}{\alpha}\right) q(z)=\frac{1}{1+a z}+\left(\frac{1-\alpha}{\alpha}\right)(1+a z) .
$$

Thus for real number $\alpha$ such that $0<\alpha \leq 1$, we notice that $q(z)$ satisfies the condition (i) and (ii) in Theorem 5 and Theorem 6 . Therefore, we obtain the following results from Theorem 5 and Theorem 6 respectively.

Theorem 13. Let $\alpha$ be a real number such that $0<\alpha \leq 1$. If $f \in \mathcal{A}$ satisfies

$$
\begin{aligned}
(1-\alpha) \frac{z f^{\prime}(z)}{\phi(f(z))}+\alpha( & \left.1+\frac{z f^{\prime \prime}(z)}{f^{\prime}(z)}-\frac{z\left(\phi(f(z))^{\prime}\right.}{\phi(f(z))}\right) \\
& \prec(1-\alpha)(1+a z)+\frac{\alpha a z}{1+a z}, z \in \mathbb{E},
\end{aligned}
$$

then

$$
\frac{z f^{\prime}(z)}{\phi(f(z))} \prec 1+a z, \text { where } 0 \leq a<1 .
$$

Theorem 14. Let $\alpha$ be a real number such that $0<\alpha \leq 1$. If $f \in \mathcal{A}$ satisfies

$$
\begin{aligned}
(1-\alpha) \frac{z f^{\prime}(z)}{f(z)}+\alpha & \left(1+\frac{z f^{\prime \prime}(z)}{f^{\prime}(z)}-\frac{z f^{\prime}(z)}{f(z)}\right) \\
& \prec(1-\alpha)(1+a z)+\frac{\alpha a z}{1+a z}, z \in \mathbb{E},
\end{aligned}
$$

then

$$
\frac{z f^{\prime}(z)}{f(z)} \prec 1+a z, \text { where } 0 \leq a<1 \text {. }
$$


R. Brar and S. S. Billing - Applications of a Differential Subordination ...

Remark 5. When we select $\beta=1, \gamma=0$ and $q(z)=\left(\frac{1+z}{1-z}\right)^{\eta}, 0<\eta \leq 1$ in Theorem 5 and Theorem 6, a little calculation yields that

$$
1+\frac{z q^{\prime \prime}(z)}{q^{\prime}(z)}-\frac{z q^{\prime}(z)}{q(z)}=\frac{1+z^{2}}{1-z^{2}}
$$

and

$$
1+\frac{z q^{\prime \prime}(z)}{q^{\prime}(z)}-\frac{z q^{\prime}(z)}{q(z)}+\left(\frac{1-\alpha}{\alpha}\right) q(z)=\frac{1+z^{2}}{1-z^{2}}+\left(\frac{1-\alpha}{\alpha}\right)\left(\frac{1+z}{1-z}\right)^{\eta} .
$$

Thus for real number $\alpha$ such that $0<\alpha \leq 1$, we notice that $q(z)$ satisfies the condition (i) and (ii) in Theorem 5 and Theorem 6 . Therefore, we have the following results from Theorem 5 and Theorem 6 respectively.

Theorem 15. Let $\alpha$ be a real number such that $0<\alpha \leq 1$. If $f \in \mathcal{A}$ satisfies

$$
\begin{aligned}
(1-\alpha) \frac{z f^{\prime}(z)}{\phi(f(z))}+\alpha & \left(1+\frac{z f^{\prime \prime}(z)}{f^{\prime}(z)}-\frac{z\left(\phi(f(z))^{\prime}\right.}{\phi(f(z))}\right) \\
& \prec(1-\alpha)\left(\frac{1+z}{1-z}\right)^{\eta}+\frac{2 \eta \alpha z}{1-z^{2}}, z \in \mathbb{E},
\end{aligned}
$$

then

$$
\frac{z f^{\prime}(z)}{\phi(f(z))} \prec\left(\frac{1+z}{1-z}\right)^{\eta} \text {, where } 0<\eta \leq 1 \text {. }
$$

Theorem 16. Let $\alpha$ be a real number such that $0<\alpha \leq 1$. If $f \in \mathcal{A}$ satisfies

$$
\begin{aligned}
(1-\alpha) \frac{z f^{\prime}(z)}{f(z)}+\alpha & \left(1+\frac{z f^{\prime \prime}(z)}{f^{\prime}(z)}-\frac{z f^{\prime}(z)}{f(z)}\right) \\
& \prec(1-\alpha)\left(\frac{1+z}{1-z}\right)^{\eta}+\frac{2 \eta \alpha z}{1-z^{2}}, z \in \mathbb{E},
\end{aligned}
$$

then

$$
\frac{z f^{\prime}(z)}{f(z)} \prec\left(\frac{1+z}{1-z}\right)^{\eta} \text {, where } 0<\eta \leq 1 \text {. }
$$

Remark 6. When we select $\beta=1, \gamma=0$ and $q(z)=\frac{\alpha^{\prime}(1-z)}{\alpha^{\prime}-z}, \alpha^{\prime}>1$ in Theorem 5 and Theorem 6, a little calculation yields that

$$
1+\frac{z q^{\prime \prime}(z)}{q^{\prime}(z)}-\frac{z q^{\prime}(z)}{q(z)}=\frac{1}{1-z}+\frac{z}{\alpha^{\prime}-z}
$$


R. Brar and S. S. Billing - Applications of a Differential Subordination ...

and

$$
1+\frac{z q^{\prime \prime}(z)}{q^{\prime}(z)}-\frac{z q^{\prime}(z)}{q(z)}+\left(\frac{1-\alpha}{\alpha}\right) q(z)=\frac{1}{1-z}+\frac{z}{\alpha^{\prime}-z}+\left(\frac{1-\alpha}{\alpha}\right)\left[\frac{\alpha^{\prime}(1-z)}{\alpha^{\prime}-z}\right] .
$$

Thus for real number $\alpha$ such that $0<\alpha \leq 1$, we notice that $q(z)$ satisfies the condition (i) and (ii) in Theorem 5 and Theorem 6. Therefore, we get the following results from Theorem 5 and Theorem 6 respectively.

Theorem 17. Let $\alpha$ be a real number such that $0<\alpha \leq 1$. If $f \in \mathcal{A}$ satisfies

$$
\begin{aligned}
(1-\alpha) \frac{z f^{\prime}(z)}{\phi(f(z))}+ & \alpha\left(1+\frac{z f^{\prime \prime}(z)}{f^{\prime}(z)}-\frac{z\left(\phi(f(z))^{\prime}\right.}{\phi(f(z))}\right) \\
& \prec(1-\alpha) \frac{\alpha^{\prime}(1-z)}{\alpha^{\prime}-z}+\frac{\left(1-\alpha^{\prime}\right) \alpha z}{(1-z)\left(\alpha^{\prime}-z\right)}, z \in \mathbb{E},
\end{aligned}
$$

then

$$
\frac{z f^{\prime}(z)}{\phi(f(z))} \prec \frac{\alpha^{\prime}(1-z)}{\alpha^{\prime}-z} \text {, where } \alpha^{\prime}>1 \text {. }
$$

Theorem 18. Let $\alpha$ be a real number such that $0<\alpha \leq 1$. If $f \in \mathcal{A}$ satisfies

$$
\begin{aligned}
(1-\alpha) \frac{z f^{\prime}(z)}{f(z)} & +\alpha\left(1+\frac{z f^{\prime \prime}(z)}{f^{\prime}(z)}-\frac{z f^{\prime}(z)}{f(z)}\right) \\
& \prec(1-\alpha) \frac{\alpha^{\prime}(1-z)}{\alpha^{\prime}-z}+\frac{\left(1-\alpha^{\prime}\right) \alpha z}{(1-z)\left(\alpha^{\prime}-z\right)}, z \in \mathbb{E},
\end{aligned}
$$

then

$$
\frac{z f^{\prime}(z)}{f(z)} \prec \frac{\alpha^{\prime}(1-z)}{\alpha^{\prime}-z}, \text { where } \alpha^{\prime}>1
$$

Remark 7. When we select $\beta=1, \gamma=1$ and $q(z)=1+\frac{2}{\pi^{2}}\left(\log \left(\frac{1+\sqrt{z}}{1-\sqrt{z}}\right)\right)^{2}$ in Theorem 5 and Theorem 6, a little calculation yields that

$$
1+\frac{z q^{\prime \prime}(z)}{q^{\prime}(z)}=\frac{1+z}{2(1-z)}+\frac{\sqrt{z}}{(1-z) \log \left(\frac{1+\sqrt{z}}{1-\sqrt{z}}\right)}
$$

and

$$
1+\frac{z q^{\prime \prime}(z)}{q^{\prime}(z)}+\frac{1}{\alpha}=\frac{1+z}{2(1-z)}+\frac{\sqrt{z}}{(1-z) \log \left(\frac{1+\sqrt{z}}{1-\sqrt{z}}\right)}+\frac{1}{\alpha} .
$$

Thus for positive real number $\alpha$, we notice that $q(z)$ satisfies the condition (i) and (ii) in Theorem 5 and Theorem 6. Therefore, we obtain the following results from Theorem 5 and Theorem 6 respectively. 
R. Brar and S. S. Billing - Applications of a Differential Subordination ...

Theorem 19. Let $\alpha$ be a positive real number. If $f \in \mathcal{A}$ satisfies

$$
\begin{aligned}
\frac{z f^{\prime}(z)}{\phi(f(z))}+\alpha & \left(\frac{z f^{\prime}(z)}{\phi(f(z))}\right)\left(1+\frac{z f^{\prime \prime}(z)}{f^{\prime}(z)}-\frac{z\left(\phi(f(z))^{\prime}\right.}{\phi(f(z))}\right) \\
& \prec 1+\frac{2}{\pi^{2}}\left(\log \left(\frac{1+\sqrt{z}}{1-\sqrt{z}}\right)\right)^{2}+\frac{4 \alpha \sqrt{z}}{\pi^{2}(1-z)} \log \left(\frac{1+\sqrt{z}}{1-\sqrt{z}}\right),
\end{aligned}
$$

then

$$
\frac{z f^{\prime}(z)}{\phi(f(z))} \prec 1+\frac{2}{\pi^{2}}\left(\log \left(\frac{1+\sqrt{z}}{1-\sqrt{z}}\right)\right)^{2}, \quad z \in \mathbb{E} .
$$

Theorem 20. Let $\alpha$ be a positive real number. If $f \in \mathcal{A}$ satisfies

$$
\begin{aligned}
& \frac{z f^{\prime}(z)}{f(z)}+\alpha\left(\frac{z f^{\prime}(z)}{f(z)}\right)(\left.+\frac{z f^{\prime \prime}(z)}{f^{\prime}(z)}-\frac{z f^{\prime}(z)}{f(z)}\right) \\
& \prec 1+\frac{2}{\pi^{2}}\left(\log \left(\frac{1+\sqrt{z}}{1-\sqrt{z}}\right)\right)^{2} \\
&+\frac{4 \alpha \sqrt{z}}{\pi^{2}(1-z)} \log \left(\frac{1+\sqrt{z}}{1-\sqrt{z}}\right)
\end{aligned}
$$

then

$$
\frac{z f^{\prime}(z)}{f(z)} \prec 1+\frac{2}{\pi^{2}}\left(\log \left(\frac{1+\sqrt{z}}{1-\sqrt{z}}\right)\right)^{2}, \quad z \in \mathbb{E} .
$$

Remark 8. When we select $\beta=1, \gamma=1$ and $q(z)=\frac{1+(1-2 \delta) z}{1-z}$, for $0 \leq \delta<$ 1 in Theorem 5 and Theorem 6, a little calculation yields that

$$
1+\frac{z q^{\prime \prime}(z)}{q^{\prime}(z)}=\frac{1+z}{1-z}
$$

and

$$
1+\frac{z q^{\prime \prime}(z)}{q^{\prime}(z)}+\frac{1}{\alpha}=\frac{1+z}{1-z}+\frac{1}{\alpha} .
$$

Thus for positive real number $\alpha$, we notice that $q(z)$ satisfies the condition (i) and (ii) in Theorem 5 and Theorem 6. Therefore, we arrive at the following results from Theorem 5 and Theorem 6 respectively.

Theorem 21. Let $\alpha$ be a positive real number. If $f \in \mathcal{A}$ satisfies

$$
\frac{z f^{\prime}(z)}{\phi(f(z))}+\alpha\left(\frac{z f^{\prime}(z)}{\phi(f(z))}\right)\left(1+\frac{z f^{\prime \prime}(z)}{f^{\prime}(z)}-\frac{z\left(\phi(f(z))^{\prime}\right.}{\phi(f(z))}\right)
$$


R. Brar and S. S. Billing - Applications of a Differential Subordination ...

$$
\prec \frac{1+(1-2 \delta) z}{1-z}+\frac{2 \alpha z(1-\delta)}{(1-z)^{2}}
$$

then

$$
\frac{z f^{\prime}(z)}{\phi(f(z))} \prec \frac{1+(1-2 \delta) z}{1-z}, \quad z \in \mathbb{E} \text {, where } 0 \leq \delta<1 .
$$

Theorem 22. Let $\alpha$ be a positive real number. If $f \in \mathcal{A}$ satisfies

$$
\begin{aligned}
\frac{z f^{\prime}(z)}{f(z)}+\alpha\left(\frac{z f^{\prime}(z)}{f(z)}\right) & \left(1+\frac{z f^{\prime \prime}(z)}{f^{\prime}(z)}-\frac{z f^{\prime}(z)}{f(z)}\right) \\
& \prec \frac{1+(1-2 \delta) z}{1-z}+\frac{2 \alpha z(1-\delta)}{(1-z)^{2}}
\end{aligned}
$$

then

$$
\frac{z f^{\prime}(z)}{f(z)} \prec \frac{1+(1-2 \delta) z}{1-z}, \quad z \in \mathbb{E} \text {, where } 0 \leq \delta<1 .
$$

Remark 9. When we select $\beta=1, \gamma=1$ and $q(z)=e^{z}$, in Theorem 5 and Theorem 6 , a little calculation yields that

$$
1+\frac{z q^{\prime \prime}(z)}{q^{\prime}(z)}=1+z
$$

and

$$
1+\frac{z q^{\prime \prime}(z)}{q^{\prime}(z)}+\frac{1}{\alpha}=1+z+\frac{1}{\alpha} .
$$

Thus for positive real number $\alpha$, we notice that $q(z)$ satisfies the condition (i) and (ii) in Theorem 5 and Theorem 6. Therefore, we have the following results from Theorem 5 and Theorem 6 respectively.

Theorem 23. Let $\alpha$ be a positive real number. If $f \in \mathcal{A}$ satisfies

$$
\frac{z f^{\prime}(z)}{\phi(f(z))}+\alpha\left(\frac{z f^{\prime}(z)}{\phi(f(z))}\right)\left(1+\frac{z f^{\prime \prime}(z)}{f^{\prime}(z)}-\frac{z\left(\phi(f(z))^{\prime}\right.}{\phi(f(z))}\right) \prec e^{z}(1+\alpha z),
$$

then

$$
\frac{z f^{\prime}(z)}{\phi(f(z))} \prec e^{z}, \quad z \in \mathbb{E} .
$$

Theorem 24. Let $\alpha$ be a positive real number. If $f \in \mathcal{A}$ satisfies

$$
\frac{z f^{\prime}(z)}{f(z)}+\alpha\left(\frac{z f^{\prime}(z)}{f(z)}\right)\left(1+\frac{z f^{\prime \prime}(z)}{f^{\prime}(z)}-\frac{z f^{\prime}(z)}{f(z)}\right) \prec e^{z}(1+\alpha z),
$$

then

$$
\frac{z f^{\prime}(z)}{f(z)} \prec e^{z}, \quad z \in \mathbb{E}
$$


Remark 10. When we select $\beta=1, \gamma=1$ and $q(z)=\frac{\alpha^{\prime}(1-z)}{\alpha^{\prime}-z}, 1 \leq \alpha^{\prime}<\frac{3}{2}$ in Theorem 5 and Theorem 6, a little calculation yields that

$$
\begin{gathered}
1+\frac{z q^{\prime \prime}(z)}{q^{\prime}(z)}=\frac{\alpha^{\prime}+z}{\alpha^{\prime}-z}, \\
1+\frac{z q^{\prime \prime}(z)}{q^{\prime}(z)}+\frac{1}{\alpha}=\frac{\alpha^{\prime}+z}{\alpha^{\prime}-z}+\frac{1}{\alpha} .
\end{gathered}
$$

For a positive real number $\alpha$, we see that $q(z)$ satisfies the conditions (i) and (ii) of Theorem 5 and Theorem 6 . Therefore, we get the following results from Theorem 5 and Theorem 6 respectively.

Theorem 25. Let $\alpha$ be a positive real number. If $f \in \mathcal{A}$ satisfies

$$
\begin{aligned}
\frac{z f^{\prime}(z)}{\phi(f(z))}+\alpha & \left(\frac{z f^{\prime}(z)}{\phi(f(z))}\right)\left(1+\frac{z f^{\prime \prime}(z)}{f^{\prime}(z)}-\frac{z\left(\phi(f(z))^{\prime}\right.}{\phi(f(z))}\right) \\
\prec & \frac{\alpha^{\prime}(1-z)}{\alpha^{\prime}-z}+\alpha \frac{\left(\alpha^{\prime}-\alpha^{\prime 2}\right) z}{\left(\alpha^{\prime}-z\right)^{2}},
\end{aligned}
$$

then

$$
\frac{z f^{\prime}(z)}{\phi(f(z))} \prec \frac{\alpha^{\prime}(1-z)}{\alpha^{\prime}-z}, \quad z \in \mathbb{E} \text {, where } 1 \leq \alpha^{\prime}<\frac{3}{2} .
$$

Theorem 26. Let $\alpha$ be a positive real number. If $f \in \mathcal{A}$ satisfies

$$
\begin{aligned}
\frac{z f^{\prime}(z)}{f(z)}+ & \alpha\left(\frac{z f^{\prime}(z)}{f(z)}\right)\left(1+\frac{z f^{\prime \prime}(z)}{f^{\prime}(z)}-\frac{z f^{\prime}(z)}{f(z)}\right) \\
& \prec \frac{\alpha^{\prime}(1-z)}{\alpha^{\prime}-z}+\alpha \frac{\left(\alpha^{\prime}-\alpha^{\prime 2}\right) z}{\left(\alpha^{\prime}-z\right)^{2}},
\end{aligned}
$$

then

$$
\frac{z f^{\prime}(z)}{f(z)} \prec \frac{\alpha^{\prime}(1-z)}{\alpha^{\prime}-z}, \quad z \in \mathbb{E} \text {, where } 1 \leq \alpha^{\prime}<\frac{3}{2} .
$$

Remark 11. When we select $\beta=1, \gamma=1$ and $q(z)=1+a z, 0 \leq a<1$ in Theorem 5 and Theorem 6, a little calculation yields that

$$
\begin{gathered}
1+\frac{z q^{\prime \prime}(z)}{q^{\prime}(z)}=1, \\
1+\frac{z q^{\prime \prime}(z)}{q^{\prime}(z)}+\frac{1}{\alpha}=1+\frac{1}{\alpha} .
\end{gathered}
$$

For a positive real number $\alpha$, we see that $q(z)$ satisfies the conditions (i) and (ii) of Theorem 5 and Theorem 6 . Therefore, we derive next two results from Theorem 5 and Theorem 6 respectively. 
R. Brar and S. S. Billing - Applications of a Differential Subordination ...

Theorem 27. Let $\alpha$ be a positive real number. If $f \in \mathcal{A}$ satisfies

$$
\frac{z f^{\prime}(z)}{\phi(f(z))}+\alpha\left(\frac{z f^{\prime}(z)}{\phi(f(z))}\right)\left(1+\frac{z f^{\prime \prime}(z)}{f^{\prime}(z)}-\frac{z\left(\phi(f(z))^{\prime}\right.}{\phi(f(z))}\right) \prec 1+a z+\alpha a z,
$$

then

$$
\frac{z f^{\prime}(z)}{\phi(f(z))} \prec 1+a z, \quad z \in \mathbb{E} \text {, where } 0 \leq a<1 .
$$

Theorem 28. Let $\alpha$ be a positive real number. If $f \in \mathcal{A}$ satisfies

$$
\frac{z f^{\prime}(z)}{f(z)}+\alpha\left(\frac{z f^{\prime}(z)}{f(z)}\right)\left(1+\frac{z f^{\prime \prime}(z)}{f^{\prime}(z)}-\frac{z f^{\prime}(z)}{f(z)}\right) \prec 1+a z+\alpha a z,
$$

then

$$
\frac{z f^{\prime}(z)}{f(z)} \prec 1+a z, \quad z \in \mathbb{E} \text {, where } 0 \leq a<1 \text {. }
$$

Remark 12. When we select $\beta=1, \gamma=1$ and $q(z)=\left(\frac{1+z}{1-z}\right)^{\eta}, 0<\eta \leq 1$ in Theorem 5 and Theorem 6, a little calculation yields that

$$
\begin{gathered}
1+\frac{z q^{\prime \prime}(z)}{q^{\prime}(z)}=\frac{1+z^{2}+2 \eta z}{1-z^{2}}, \\
1+\frac{z q^{\prime \prime}(z)}{q^{\prime}(z)}+\frac{1}{\alpha}=\frac{1+z^{2}+2 \eta z}{1-z^{2}}+\frac{1}{\alpha} .
\end{gathered}
$$

For a positive real number $\alpha$, we see that $q(z)$ satisfies the conditions (i) and (ii) of Theorem 5 and Theorem 6 . Therefore, we obtain the following results from Theorem 5 and Theorem 6 respectively.

Theorem 29. Let $\alpha$ be a positive real number. If $f \in \mathcal{A}$ satisfies

$$
\begin{aligned}
& \frac{z f^{\prime}(z)}{\phi(f(z))}+\alpha\left(\frac{z f^{\prime}(z)}{\phi(f(z))}\right)\left(1+\frac{z f^{\prime \prime}(z)}{f^{\prime}(z)}-\frac{z\left(\phi(f(z))^{\prime}\right.}{\phi(f(z))}\right) \\
& \prec\left(\frac{1+z}{1-z}\right)^{\eta}\left[1+\frac{2 \alpha \eta z}{1-z^{2}}\right],
\end{aligned}
$$

then

$$
\frac{z f^{\prime}(z)}{\phi(f(z))} \prec\left(\frac{1+z}{1-z}\right)^{\eta}, \quad z \in \mathbb{E} \text {, where } 0<\eta \leq 1 \text {. }
$$


R. Brar and S. S. Billing - Applications of a Differential Subordination ...

Theorem 30. Let $\alpha$ be a positive real number. If $f \in \mathcal{A}$ satisfies

$$
\begin{aligned}
\frac{z f^{\prime}(z)}{f(z)}+ & \alpha\left(\frac{z f^{\prime}(z)}{f(z)}\right)\left(1+\frac{z f^{\prime \prime}(z)}{f^{\prime}(z)}-\frac{z f^{\prime}(z)}{f(z)}\right) \\
& \prec\left(\frac{1+z}{1-z}\right)^{\eta}\left[1+\frac{2 \alpha \eta z}{1-z^{2}}\right],
\end{aligned}
$$

then

$$
\frac{z f^{\prime}(z)}{f(z)} \prec\left(\frac{1+z}{1-z}\right)^{\eta}, \quad z \in \mathbb{E} \text {, where } 0<\eta \leq 1 \text {. }
$$

\section{REFERENCES}

[1] L. Brickman, $\phi$-like analytic functions, I, Bull. Amer. Math. Soc., 79 (1973), $555-558$.

[2] W. C. Ma and D. Minda, Uniformly convex functions, Ann. Polon. Math. 57, 2 (1992), 165-175.

[3] S. S. Miller and P. T. Mocanu, Differential subordination Theory and applications, Marcel Dekker New York and Basel. (2000).

[4] V. Ravichandran, N. Magesh and R. Rajalakshmi, On certain applications of differential subordinations for $\phi$-like functions, Tamkang J. Math., 36, 2 (2005), 137-142.

[5] F. Ronning, Uniformly convex functions and a corresponding class of starlike functions, Proc. Amer. Math. Soc. 118, 1 (1993), 189-196.

[6] St. Ruscheweyh, A subordination theorem for $\phi$-like functions, J. London Math. Soc., 2, 13 (1976), 275-280.

[7] T. N. Shanmugam, S. Sivasubramanian and Maslina Darus, Subordination and superordination results for $\phi$-like functions, J. Inq. Pure and App. Math., 8, 1 (2007), Art. 20, 6pp.

Richa Brar

Department of Mathematics,

Sri Guru Granth sahib World University,

Fatehgarh Sahib - 140407, Punjab

email: richabrar4@gmail.com

S. S. Billing

Department of Mathematics, 
R. Brar and S. S. Billing - Applications of a Differential Subordination ...

Sri Guru Granth sahib World University, Fatehgarh Sahib - 140407, Punjab email: ssbilling@gmail.com 